\title{
Evaluation of Synthesized Nanohydroxyapatite-Nanocellulose Composites as Biocompatible Scaffolds for Applications in Bone Tissue Engineering
}

\author{
Claudia S. Herdocia-Lluberes, ${ }^{1,2}$ Simara Laboy-López, ${ }^{2,3}$ Stefannie Morales, ${ }^{2}$ \\ Tania J. Gonzalez-Robles, ${ }^{2,3}$ José A. González-Feliciano, ${ }^{2}$ and Eduardo Nicolau ${ }^{2,3}$ \\ ${ }^{1}$ Department of Biology, University of Puerto Rico, Rio Piedras Campus, P.O. Box 23346, San Juan, PR 00931-3346, USA \\ ${ }^{2}$ Molecular Science Research Center, University of Puerto Rico, Suite 2, 1390 Ponce De Leon Avenue, San Juan, PR 00931-3346, USA \\ ${ }^{3}$ Department of Chemistry, University of Puerto Rico, Rio Piedras Campus, P.O. Box 23346, San Juan, PR 00931-3346, USA
}

Correspondence should be addressed to Eduardo Nicolau; eduardo.nicolau@upr.edu

Received 26 September 2015; Accepted 30 November 2015

Academic Editor: Tae-Yub Kwon

Copyright (c) 2015 Claudia S. Herdocia-Lluberes et al. This is an open access article distributed under the Creative Commons Attribution License, which permits unrestricted use, distribution, and reproduction in any medium, provided the original work is properly cited.

\begin{abstract}
Basic calcium phosphate (BCP) crystals have been associated with many diseases due to their activation of signaling pathways that lead to their mineralization and deposition in intra-articular and periarticular locations in the bones. In this study, hydroxyapatite (HAp) has been placed in a polysaccharide network as a strategy to minimize this deposition. This research consisted of the evaluation of varying proportions of the polysaccharide network, cellulose nanocrystals (CNCs), and HAp synthesized via a simple sol-gel method. The resulting biocompatible composites were extensively characterized by means of thermogravimetric analysis (TGA), powder X-ray diffraction (XRD), Fourier transform infrared spectroscopy (FT-IR), dynamic light scattering (DLS), zeta potential, and scanning electron microscopy (SEM). It was found that an nHAp $=$ CNC ratio presented greater homogeneity in the size and distribution of the nanoparticles without compromising the crystalline structure. Also, incorporation of bone morphogenetic protein 2 (BMP-2) was performed to evaluate the effects that this interaction would have in the constructs. Finally, the osteoblast cell (hFOB 1.19) viability assay was executed and it showed that all of the materials promoted greater cell proliferation while the nHAp > CNC proportion with the inclusion of the BMP-2 protein was the best composite for the purpose of this study.
\end{abstract}

\section{Introduction}

Advances in Bone Tissue Engineering (BTE) hold promise for the development of new functional coatings for bone regeneration. The integration of novel bionanomaterials that induce bone regeneration can contribute to the field of BTE and help settle the incidence of bone disorders and conditions $[1,2]$. BTE has been a field of study of increasing interest over the past few decades and it focuses on alternative treatment options that will ideally eliminate issues of current clinically used treatments with bone implants, such as donor site morbidity, immune rejection, and pathogen transfer. The main goal of this field is to create bone grafts that enhance bone repair and regeneration of damaged tissue, combining body cells with highly porous scaffold bionanomaterials [2]. Scaffolds are structures that bring support to the bone by promoting biocompatibility that enriches with nutrients the existing natural bone; therefore, it must imitate its composition in the best way possible. Scaffolds must be stressshielding, biocompatible, and porous structures that resemble the bone $[3,4]$. The most studied material to replace and regenerate human hard tissue is hydroxyapatite (HAp), an interesting biomaterial with potential to improve the field of medicine and dentistry. Hydroxyapatite $\left(\mathrm{Ca}_{10}\left(\mathrm{PO}_{4}\right)_{6}(\mathrm{OH})_{2}\right.$, HAp) is chemically and structurally similar to the inorganic components of bone, enamel, and dentin; it is also bioactive and supports bone ingrowth and osseointegration. Due to these unique features HAp has gained recognition and 
importance in the field of BTE. It has also been successfully used as bone filler, aesthetic restorative, coating of orthopedic implants, filler of inorganic/polymer composites, and cellculture carrier, among others [5].

Overall, the use of basic calcium phosphate (BCP) crystals, such as HAp, has been associated with many diseases due to their deposition in intra-articular and periarticular locations site [6]. New pathogenic mechanisms for the deposition mechanisms of BCPs suggest that the extracellular inorganic phosphate, inorganic pyrophosphate, and $\mathrm{Ca}^{2+}$ concentrations are critical determinants of mineralization $[7,8]$. It has been found that BCP crystals activate three major signaling pathways: crystal endocytosis and intracellular crystal dissolution with subsequent intracellular calcium increase [9]. Therefore, placing the HAp in a polysaccharide network might prevent this cell activation. Additionally, the use of HAp as a coating agent for metallic implants has been discouraged by many scientists because it is limited to nonload-bearing applications due to the disproportion between the different Young modulus of each. This is an undesired effect because it leads to bone density loss and even fracture or disconnection of bone from the implant. It is of our interest that the polysaccharide network could serve as a hardening intermediate between the bone and the implant [10].

There are many polysaccharides that have been used for tissue engineering applications such as chitosan and gelatin $[11,12]$. A similar material, cellulose nanocrystals (CNCs), has been the subject of study in several applications because it is one of the most abundant biopolymers on earth; it is nontoxic and has a Young modulus of $167.5 \mathrm{GPa}$ comparable to the stiffness of titanium, copper, and bronze materials used in bone implants [13]. CNCs are the ordered crystalline regions of cellulose microfibers (cellulose molecule chains connected through hydrogen bonding with approximately $5-50 \mathrm{~nm}$ in diameter) which can be obtained from hydrolysis $[14,15]$.

Due to all of the above, herein we propose to synthesize nanohydroxyapatite (nHAp) - nanocellulose (CNC) composites via the sol-gel route in order to develop uniform molecular-level mixing of the calcium and phosphorus precursors. This strategy might provide several advantages such as improving chemical homogeneity of the resulting HAp, low synthesis temperature, and promotion of controlled growth of spherical nanoparticles [16, 17]. The sol-gel product is recognized by the nanometric dimension of the primary particles that is known to improve the contact reaction and the stability at the artificial/natural bone interface $[16,18]$. In the present paper we report the synthesis and characterization of nHAp with the incorporation of CNCs via the sol-gel method and its characterization using different proportions of the bionanomaterials for the creation of scaffolds that will contribute to the field of BTE. Through the evaluation of different proportions of the HAp and CNC it was possible to determine which proportion promoted a better synthesis of nHAp nanoparticles in terms of homogeneity and dispersion. We present the addition of bone morphogenetic protein (BMP2) to the synthesized composites as it is an important protein involved in the development and regeneration of tissue and cartilage which makes it promising for our purpose [19].
Finally, we present the inclusion of osteoblast cells into the nanoconstructs with BMP-2 in order to determine the biocompatibility of these materials. Nanomaterials have proven to be noncytotoxic in previous studies [20-22].

\section{Materials and Methods}

2.1. Materials. Cellulose nanocrystals (CNCs) $11.8 \%$ aqueous solution was purchased from the University of Maine Process and Development Center (Orono, USA). Hydroxyapatite (HAp), ethanol, acetic acid, phosphate buffered solution $1 \mathrm{x}$, $\mathrm{pH}=7.2$ (PBS), and bone morphogenetic protein 2 (BMP2) were purchased from Sigma-Aldrich. All chemicals and solvents were used as received without further purification. Deionized water (18.3 MW, MilliQ Direct 16) was used at all times.

\subsection{Synthesis of Hydroxyapatite Nanoparticles with Cellulose} Nanocrystals ( $\mathrm{HA}$ H-CNC) Composites. In order to compare the effects of polysaccharide to BCD crystals, three composites were prepared with different proportions of HAp and CNC. The three compounds were prepared, following the proportions of Hap > CNC, Hap = CNC, and Hap < CNC. The same ratio of ethanol and acetic acid was used to produce nanohydroxyapatite (nHAp), following a similar procedure to that of Monreal Romero et al. [23]. In brief, $1 \mathrm{~mL}$ of $99 \%$ chemically pure grade biopolymer $\beta-1,4-\mathrm{D}$ linked glucose (CNC) at $3.2 \%$ wt was placed at $30^{\circ} \mathrm{C}$ for 30 minutes and added to the solutions of HAp with $5 \mathrm{~mL}$ ethanol and $1.25 \mathrm{~mL}$ acetic acid for gel formation. The first solution (nHAp > CNC) was prepared using $0.5 \mathrm{~g}$ HAp and $9.17 \mu \mathrm{L}$ CNCs $(3.2 \%)$, the second solution $(\mathrm{nHAp}=\mathrm{CNC})$ consisted of $0.5 \mathrm{~g}$ HAp and $458 \mu \mathrm{L} \mathrm{CNCs} \mathrm{(3.2 \% ),} \mathrm{and} \mathrm{the} \mathrm{third} \mathrm{solution}$ (nHAp < CNC) consisted of $0.01 \mathrm{~g}$ HAp and $458 \mu \mathrm{L} \mathrm{CNCs}$ (3.2\%); subsequently the solutions were sonicated at $30^{\circ} \mathrm{C}$ for 15 minutes. After sonication each gel was placed in a $50 \mathrm{~mL}$ tube and centrifuged 3 times at 12,500 rpm for 5 minutes at $30^{\circ} \mathrm{C}$; in-between the procedure, the concentrated gel was poured off and the precipitate was resuspended twice with deionized water to eliminate any amount of gel residue. The resulting pellet was freeze-dried for 24 hours and stored for further characterization.

2.3. Inclusion of BMP-2 to $n H A p$. For the inclusion of BMP-2 to the composites, $10 \mu \mathrm{g}$ of BMP-2 was diluted in $1000 \mu \mathrm{L}$ of PBS first. Afterwards, two solutions of $0.01 \mathrm{~g}$ of nHAp $>\mathrm{CNC}$ and $n H A p=C N C$ were prepared in $900 \mu \mathrm{L}$ of PBS and $100 \mu \mathrm{L}$ of the solution containing the BMP-2 was added to each.

2.4. Cell Proliferation and Viability Assay. Human osteoblast cell line (hFOB 1.19) was purchased from the American Type Culture Collection (Manassas, VA, USA). Osteoblast cells were cultured in 1:1 mixture of Ham's F12 Medium/Dulbecco's Modified Eagle's Medium (Gibco by Life Technologies) supplemented with $2.5 \mathrm{mM}$ L-glutamine, $10 \%$ fetal bovine serum (Gibco by Life Technologies), and $0.3 \mathrm{mg} / \mathrm{mL}$ G148 (Gibco by Life Technologies) at $34^{\circ} \mathrm{C}$ with $5 \% \mathrm{CO}_{2}$. To assess the cell viability effect of HAp, CNC, Hap > 
CNC, Hap $=$ CNC, Hap $>$ CNC + BMP -2 , and Hap $=\mathrm{CNC}+$ BMP-2 nanoparticles, the MTS CellTiter 96 AQueous Solution Cell proliferation Assay (Promega) was used. In brief, cells were plated at $7.5 \times 10^{4}$ cells $/ \mathrm{mL}$ in 96 -well plates (Falcon) in contact with $5 \mathrm{mg} / \mathrm{mL}$ of the different nanoconstructs and incubated for $24 \mathrm{hrs}$. Then, $20 \mu \mathrm{L}$ of MTS reagent was added and cells were incubated for $1 \mathrm{hr}$. Thereafter, the 96-well plates were centrifuged at $1000 \mathrm{rpm}$ for $10 \mathrm{~min}$ to avoid any further light scattering from the nanomaterials. Then, $100 \mu \mathrm{L}$ of the supernatant was transferred to a new 96-well plate and absorbance was measured at $490 \mathrm{~nm}$ using a Synergy H1 Hybrid Microplate Reader from BioTek. The results were expressed as percentage (\%) of cell viability and the statistical analysis was performed with GraphPad Prism 6.

2.5. Physical Characterization of $n H A p-C N C$ Composites. In order to fully characterize the composite material, several physical characterization techniques were employed in this study.

2.5.1. Thermogravimetric Analysis (TGA). A Perkin-Elmer STA 6000 simultaneous thermal analyzer was used to measure the changes in weight and heat flow as a function of temperature. Approximately $10 \mathrm{mg}$ of each sample was added to ceramic crucible and heating of $30-750^{\circ} \mathrm{C}$ at a ramp of $20^{\circ} \mathrm{C} / \mathrm{min}$ in air atmosphere at a flow rate of $20 \mathrm{~mL} / \mathrm{min}$.

2.5.2. X-Ray Diffraction (XRD) Analysis. Powder X-ray diffraction measurements were conducted over 10 to $90^{\circ}$ $2 \theta$ range using a Rigaku SmartLab diffractometer at $40 \mathrm{kV}$ and $44 \mathrm{~mA}$ equipped with monochromatic $\mathrm{CuK} \alpha(1.54 \AA) \mathrm{X}$ ray radiation. X-ray diffraction patterns of composites were recorded in intervals of $20^{\circ} \mathrm{C}$.

\subsubsection{Dynamic Light Scattering (DLS) and Z-Potential Analy-} sis. Malvern ZetaSizer Nano Series with $4 \mathrm{~mW} 632.8 \mathrm{~nm}$ laser was used to determine the average diameter of composite suspensions. First, suspensions were sufficiently diluted with deionized water to avoid agglomeration; then approximately $1 \mathrm{~mL}$ of suspension was added to a disposable plastic cuvette. The backscattering mode was used in triplicate for all the samples and the $Z$-average (i.e., hydrodynamic radius) and polydispersity index (PDI) were recorded.

2.5.4. Scanning Electron Microscopy (SEM). Scanning electron microscopy (SEM) images were recorded using a JEOL 5800LV Scanning Microscope with electron beam energy of $20 \mathrm{kV}$. The samples were freeze-dried before measurement and, to avoid charge accumulation, a thin film of gold (15 nm) was added to the surface.

2.5.5. Infrared Spectroscopy (FTIR). Infrared spectra of freeze-dried samples were recorded on a Bruker tensor 27 Fourier transform infrared using Attenuated Total Reflectance (ATR). Sample was placed in a glass slide and then pressed using a diamond probe. The spectral width

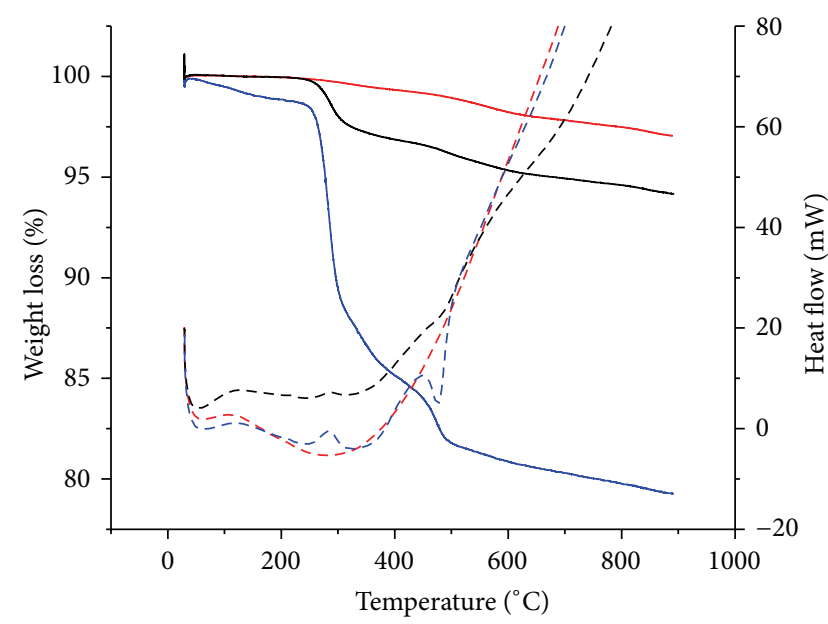

FIGURE 1: TGA and DSC analysis of nHAp $>$ CNC (red), nHAp = CNC (black), and nHAp < CNC (blue), in air atmosphere at $20 \mathrm{~mL} /$ $\min$ and a ramp of $20^{\circ} \mathrm{C} / \mathrm{min}$.

ranged from 400 to $4000 \mathrm{~cm}^{-1}$, with $4 \mathrm{~cm}^{-1}$ resolution and accumulation of 64 scans.

\section{Results and Discussion}

3.1. Physical Characterization. The main objective of this work was to prepare and characterize biocompatible constructs for Bone Tissue Engineering and repair. Nevertheless, it was of interest to extensively characterize the prepared materials to account for any structural or chemical changes in the products. In this sense, thermal stability of the material provides an indication of the interactions between particles and also about the critical transition temperatures in the material. In order to determine this, a dual thermal gravimetric analyzer with differential scanning calorimetry functions was utilized and results are shown in Figure 1. This figure shows the weight loss (straight lines) and heat flow (dotted lines) of the different nHAp-CNC constructs. From these results, a temperature drop at $c a .300^{\circ} \mathrm{C}$ was consistently observed and is ascribed to the thermal decomposition of the CNC while the nHAp decomposition transition was not observed as it belongs to a region of approximately $1080^{\circ} \mathrm{C}$, although some mass loss is present. The decomposition of nHAp occurs in three steps: (1) the appearance of $\mathrm{Ca}_{10}\left(\mathrm{PO}_{4}\right)_{6} \mathrm{O}_{x}(\mathrm{OH})_{2-2 x}$ as a transition product for the (2) dehydroxylation producing oxyapatite $\mathrm{Ca}_{10}\left(\mathrm{PO}_{4}\right)_{6} \mathrm{O}$ ending with (3) decomposition of this oxyapatite and resulting calcium phosphates [24]. The mass losses belonging to the first two steps can be observed at around $400^{\circ} \mathrm{C}$ and $600^{\circ} \mathrm{C}$, respectively, for each sample. The $\mathrm{nHAp}>\mathrm{CNC}$ sample (red) is the most stable of all, maybe due to the higher proportion of Hap, followed by the nHAp = CNC (black) with a welldefined transition belonging to the CNC and nHAP. Lastly, the nHAp $<$ CNC sample (blue) shows less thermal stability due to a lower amount of $\mathrm{CNC}$ in the sample in comparison to the nHAp with a higher change in mass loss at $300^{\circ} \mathrm{C}$ belonging to the CNC. Also, in this sample a transition at 


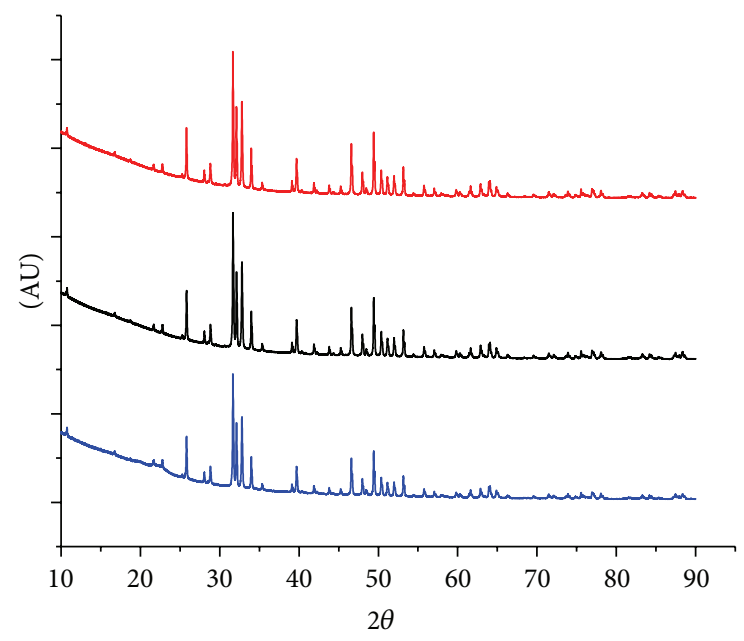

FIgURE 2: Powder XRD analysis of nHAp > CNC (red), nHAp = CNC (black), and nHAp < CNC (blue).

ca. $450^{\circ} \mathrm{C}$ ascribed to the mass loss from the first step of the decomposition of nHAp appears to be more significant. This behavior may be due to the expected stronger interaction between nHAp and CNC that will ultimately cause heat dissipation in order to reach the transition product. The heat flow results also evidence the evaporation of remaining water in the samples. The transitions ascribed to the water removal are not very noticeable in the gravimetric results at ca. $130^{\circ} \mathrm{C}$. These results suggest a correlation between the ratio of nHAp and the thermal stability. It is noticeable that the samples with higher hydroxyapatite content provide better thermal stability as it was expected due to the degradation temperature of nHAp being much higher than that of CNC.

After observing the variations in thermal stability of the constructs, it was important to determine if any crystallinity loss is observed for the prepared materials. In order to assess this, XRD was carried out and the resulting patterns are shown in Figure 2. The CNC peaks can be observed between $10^{\circ}$ and $25^{\circ}$ with characteristic phases of (110) at $17^{\circ}$ and (200) at $26^{\circ}$; a zoomed version of this area can be observed in Figure $3[25,26]$. The nHAp characteristic phases are observed at $32^{\circ}, 39^{\circ}$, and $49^{\circ}$ with peaks corresponding to the phases of (311), (410), and (313), respectively (COD ID 2300273) [26]. As a qualitative observation, the intensity of the CNC peaks is much lower than those of the nHAp due to the high crystallinity of nHAp in comparison to CNC, but this does not mean that the CNC loses crystallinity. As can be observed, all diffraction patterns showed the same behavior leading to the conclusion that the crystallinity of the sample was not compromised after sol-gel reaction with polysaccharide network. It is important to note that as the crystallinity of the cellulose decreases, the level of water absorption and biodegradability increases because with decreasing crystallinity cellulose fibers get more disorganized and hence more susceptible to degradation processes [27]. Because of this, the CNC in the synthesized composite will not degrade which sustains the initial purpose of using CNC that can serve as a hardening agent due to its high Young's

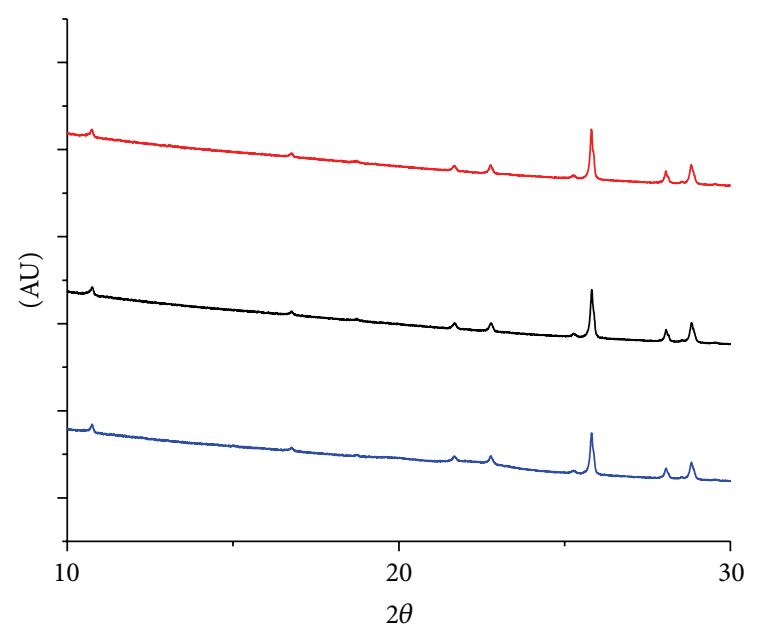

FIgure 3: Powder XRD analysis of CNC phase in nHAp > CNC (red), nHAp = CNC (black), and nHAp $<$ CNC (blue).

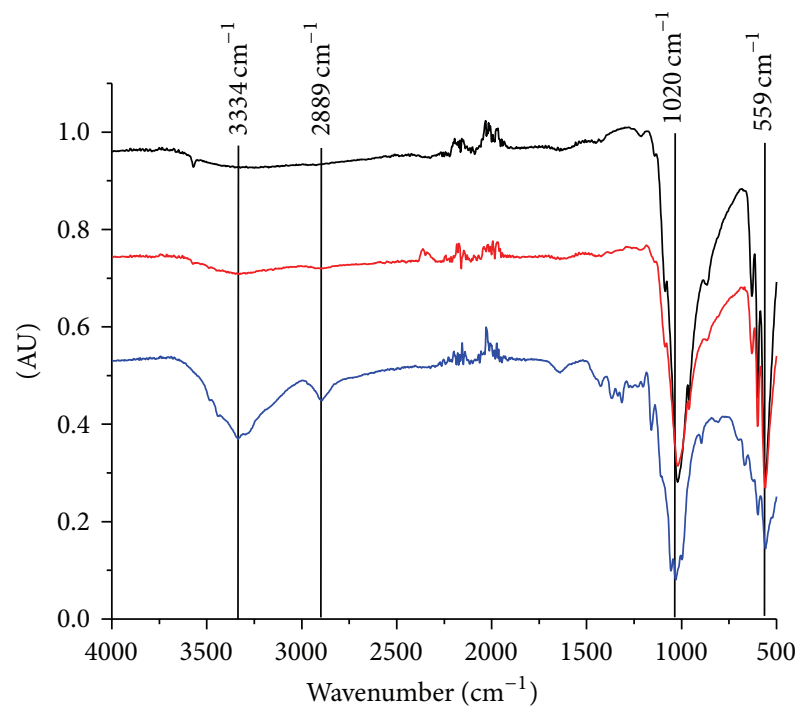

FIGURE 4: FTIR analysis of nHAp > CNC (red), nHAp = CNC (black), and nHAp < CNC (blue).

modulus and reinforcing the strength of the bone. It is also possible that this polymer network formed by cellulose may prevent the mineralization of HAp and deposition in the intra-articular and periarticular locations.

Moreover, even when the crystallinity of the samples was conserved, it was of our interest to determine any chemical changes in the surface of the composites after the synthesis. FTIR was carried out from 400 to $4000 \mathrm{~cm}^{-1}$ (Figure 4) and the presence of $\mathrm{CNC}$ is confirmed by the band at around $3400 \mathrm{~cm}^{-1}$ due to OH-stretching, the band at $1640 \mathrm{~cm}^{-1}$ likely due to the $\mathrm{OH}$-bending vibration of adsorbed water, and the band at $2889 \mathrm{~cm}^{-1}$ assigned to $\mathrm{C}-\mathrm{H}$ aliphatic stretching. Other noticeable bands from C-O-C (ether) at $1200 \mathrm{~cm}^{-1}$ and $\mathrm{CH}_{2}$ (alkaline) at $692 \mathrm{~cm}^{-1}$ are also worth noting. It is important to observe that even though nHAp is an ionic compound, its anions $\left(\mathrm{PO}_{4}{ }^{2-}\right)$ are present at $1020 \mathrm{~cm}^{-1}$ and 


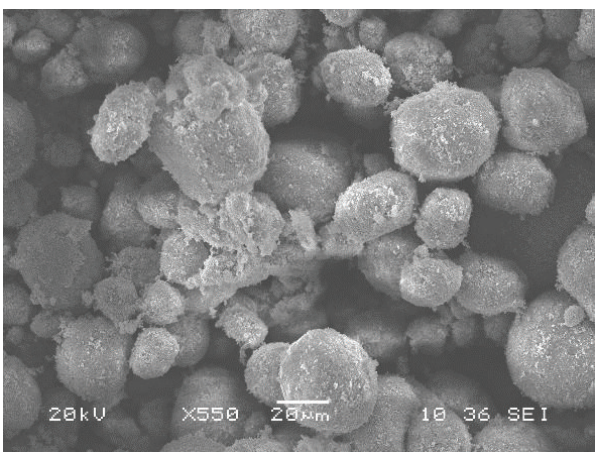

(a)

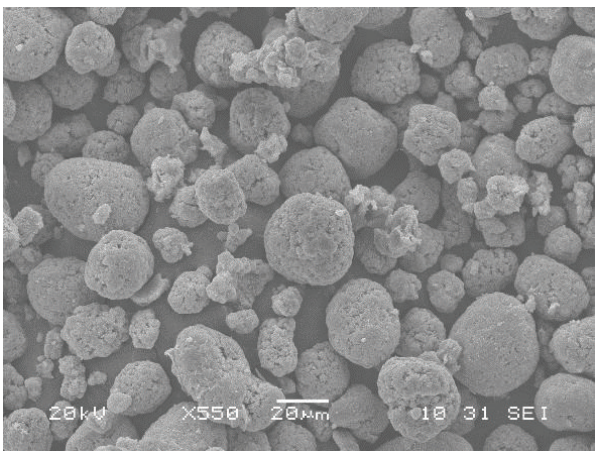

(c)

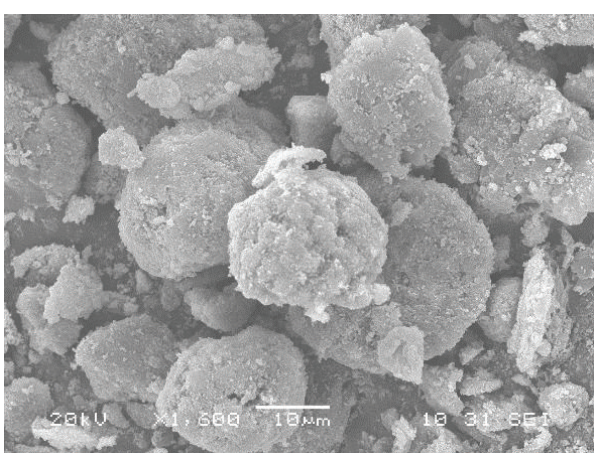

(e)

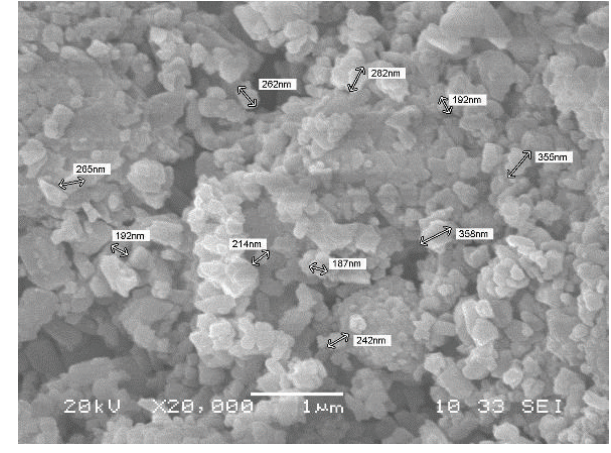

(b)

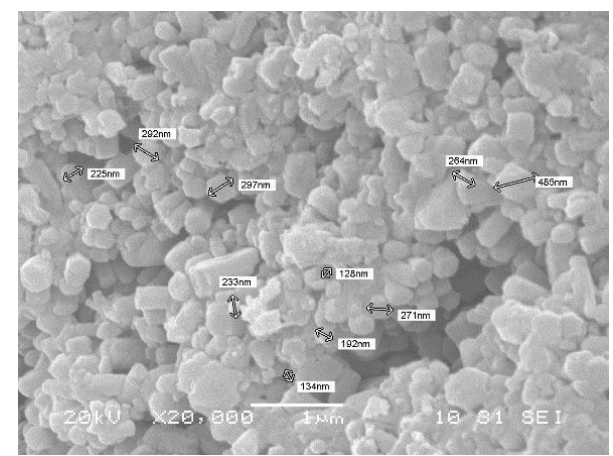

(d)

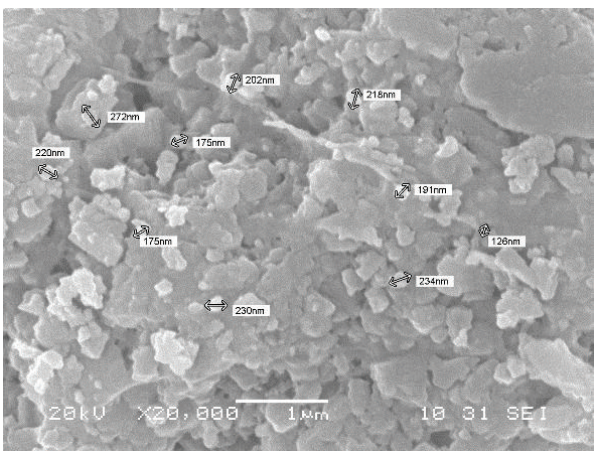

(f)

Figure 5: SEM analyses of nHAp > CNC ( $a$ and b), nHAp = CNC (c and d), and nHAp < CNC (e and f).

$559 \mathrm{~cm}^{-1}$. The peaks around $2000 \mathrm{~cm}^{-1}$ are attributed to the presence of $\mathrm{CO}_{2}$ in the samples, so these were discarded for the analysis of the spectrum. The effect of proportion is clearly visible, especially in nHAp < CNC, in the intensity of the graphs.

It has been noted that the composite size and homogeneity of the particles can influence the adherence of tissue to the host implant. As a strategy to account for the composite size, DLS analyses were conducted and are shown in Table 1. First, the size for natural HAp and CNC was determined and then the analysis of the particle size and distribution for the composites was carried out. As can be observed for the $Z$ average $\left(R_{H}\right)$ results, most particles fluctuated between 289 and $311 \mathrm{~nm}$. The results showed that the sizes for the nanoparticles were $311 \mathrm{~nm}$ for the $\mathrm{nHAP}>\mathrm{CNC}, 289 \mathrm{~nm}$ for the $\mathrm{nHAp}$ $=\mathrm{CNC}$, and $307 \mathrm{~nm}$ for the nHAp < CNC composites. The polydispersive index (PDI) for the nHAP > CNC and nHAp $=$ CNC samples was the lowest with a value of 0.21 which indicates a good size uniformity of the particles, a factor that is notably missing in the $\mathrm{nHAp}<\mathrm{CNC}$. The average reduction of particle size from starting natural HAp with an average size of $2061 \mathrm{~nm}$ is approximately $85.7 \%$, which proves that the polymer network was a determining factor in this size reduction; a representation of this interaction can be observed in Scheme 1. To further verify this, SEM was carried out to correlate the size and homogeneity of the composites and is presented in Figure 5. From these images it can be observed that the nHAp > CNC sample presents a high porosity while the $\mathrm{nHAp}=\mathrm{CNC}$ showed a better uniformity in the spherical shape, distribution, and particle size and the nHAp < CNC showed neither of these. Also, in agreement with DLS, the sizes of the particles are between 150 and $300 \mathrm{~nm}$ with 


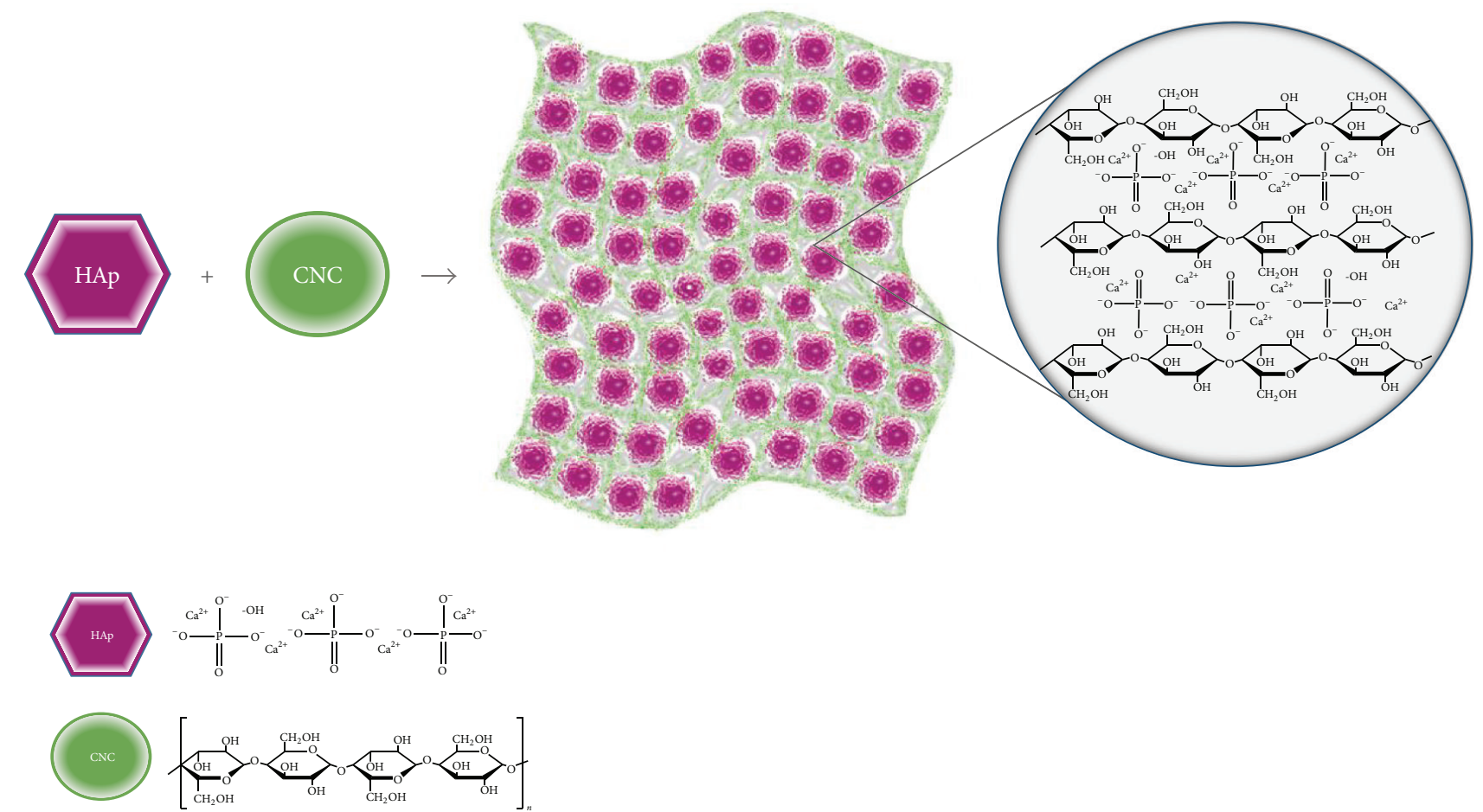

SCHEME 1: Representation of the interaction between nanohydroxyapatite (nHAp) and cellulose nanocrystals (CNCs) polymer network.

TABLE 1: DLS analyses for HAp, CNC, and nHAp-CNC at a $1 \% \mathrm{w} / \mathrm{v}$.

\begin{tabular}{lcc}
\hline Sample $^{*}$ & $Z$-average $(\mathrm{nm})$ & PDI \\
\hline nHAp > CNC (3.2\%) & $311 \pm 7$ & $0.21 \pm 0.02$ \\
nHAp = CNC (3.2\%) & $289 \pm 3$ & $0.21 \pm 0.01$ \\
nHAp < CNC (3.2\%) & $307 \pm 5$ & $0.43 \pm 0.00$ \\
Natural HAp & $1965 \pm 170$ & $0.29 \pm 0.04$ \\
CNC & $142.3 \pm 0.72$ & $0.30 \pm .007$ \\
\hline
\end{tabular}

${ }^{*}$ All samples at $\mathrm{H}_{2} \mathrm{O}(10 \mathrm{mg} / \mathrm{mL})$ at $\mathrm{pH}=7.07$.

few particles whose size is over $300 \mathrm{~nm}$. The presence of submicron particles is attributed to the aggregation factor that occurs when the solution is in suspension; nevertheless the formation of smaller nanoparticles is present. DLS was also carried out for the BMP-2 protein alone and with its incorporation to the composites, a representation of this interaction can be observed in Scheme 2. The BMP-2 size was found to be $59.97 \mathrm{~nm}$ and for the nHAp $<$ CNC and nHAp = $\mathrm{CNC}$ composites with the protein, it was observed that the size of the nanoparticles remained around $349 \mathrm{~nm}$ which is still in the range of our composites without the protein with a PDI of 0.24 (Table 2). These results in fact corroborate that the sol-gel method followed by Monreal Romero et al. is, in fact, effective for these types of materials [23].

After thorough characterization of the composites, $Z$ potential was performed in order to determine the overall charge of the nHAp > CNC and nHAp = CNC composites with and without the BMP-2 (Table 3). The analyses were run in PBS in order to understand how the composites would behave under physiological conditions. The overall
TABLE 2: DLS analyses for BMP-2, nHAp $>$ CNC, and $n H A p=C N C$ composites with protein at $1 \% \mathrm{w} / \mathrm{v}$.

\begin{tabular}{lcc}
\hline Sample $^{*}$ & $Z$-average $(\mathrm{nm})$ & PDI \\
\hline nHAp $>$ CNC $(3.2 \%)+$ BMP-2 & $333.4 \pm 11$ & $0.18 \pm 0.01$ \\
nHAp = CNC (3.2\%) + BMP-2 & $364.6 \pm 2$ & $0.30 \pm 0.02$ \\
BMP-2 & $59.97 \pm 0.9$ & $0.46 \pm 0.04$ \\
\hline${ }^{*}$ All samples in PBS at pH = 7.20. & &
\end{tabular}

TABLE 3: Zeta potential measurements of $\mathrm{nHAp}>\mathrm{CNC}$ and $\mathrm{nHAp}=$ CNC with BMP-2.

\begin{tabular}{lc}
\hline Sample & Zeta potential $(\mathrm{mV})$ \\
\hline Natural HAp & $-20 \pm 2$ \\
BMP-2 & $-11.4 \pm 0.7$ \\
nHAp > CNC & $-18 \pm 1$ \\
nHAp = CNC & $-11.0 \pm 0.6$ \\
nHAp > CNC \& BMP-2 & $-17.3 \pm 0.6$ \\
nHAp = CNC \& BMP-2 & $-20 \pm 2$ \\
\hline
\end{tabular}

${ }^{*}$ All samples at $1 \mathrm{mg} / \mathrm{mL}$ in $\mathrm{PBS}$ solvent at $\mathrm{pH}=7.2$.

charge of the HAp alone was found to have an average charge of $-19.36 \mathrm{mV}$. The isoelectric point of the BMP-2 is 8.5 and therefore below this value the BMP-2 will be positively charged [28]. When the $Z$-potential measurement was performed with the protein alone, the charge was $-12.8 \mathrm{mV}$, which indicates that there is in fact an interaction occurring between the protein and composite. A recent study about thermodynamics studies on the BMP-2 adsorption onto different hydroxyapatite surfaces conducted by $\mathrm{Lu}$ and 


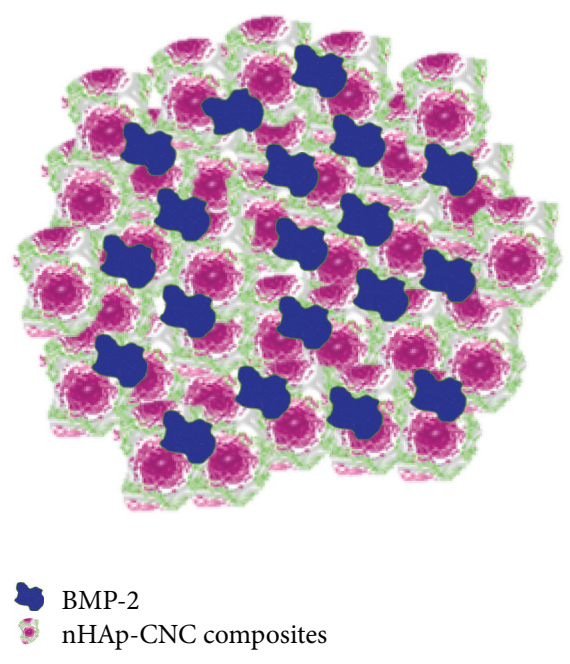

Scheme 2: Representation of the interaction between the nHAp/ $\mathrm{CNC}$ nanocomposites with the BMP-2.

team has reported that the process of adsorption is by physisorption where the initial driving force is governed by an electrostatic interaction between HAp (negatively charged at $\mathrm{pH}=7.2$ ) and BMP-2 (positively charged at $\mathrm{pH}=7.2$ ). They report that this process has a positive $\Delta H$ corresponding to an endothermic process and negative $\Delta G$ corresponding to a spontaneous and energetically favored process [29]. The composites alone had an average charge of -11.4 and $-18.1 \mathrm{mV}$ for the nHAp $>\mathrm{CNC}$ and $\mathrm{nHAp}=\mathrm{CNC}$, respectively, and the charges with the BMP-2 were lowered to -11.0 and $-17.3 \mathrm{mV}$, respectively. Several studies have already shown that the incorporation of BMP-2 results in an initial burst release followed by a stable sustained release of the protein [30]. This slight decrease in the overall charge suggests that there is in fact an interaction between the composites and the protein although this does not provide any inquiry over the stability of the construct.

3.2. Biocompatibility of Nanocomposites with Osteoblast Cells. In recent years, a great interest has been dedicated to the development of biocompatible and nontoxic nanoparticles that can be used in bone implants and biomedical devices. For instance, studies with carbon nanotubes have been shown to support the osteoblastic cells grow. However, the hydrophobic nature of carbon nanotubes requires extensive functionalization with hydrophilic conjugates in order to afford its biocompatibility [31]. Here in this study we used biocompatible nanohydroxyapatite/nanocellulose composites. To examine the effect in cell viability of such nanoconstructs, osteoblasts cells were exposed to $5 \mathrm{mg} / \mathrm{mL}$ of nHAp, CNC, nHAp > CNC, nHAp $=$ CNC, nHAp $>$ CNC + BMP-2, and nHAp $=\mathrm{CNC}+$ BMP-2 nanoparticles for $24 \mathrm{hrs}$. Then, cell viability was measured by MTS assay. The results for these experiments are shown in Figure 6 and it demonstrates that neither of the compounds compromised the osteoblast cell viability. As can be noted, the nHAp > CNC + BMP-2 composite had the highest growth of osteoblast in comparison to the others.

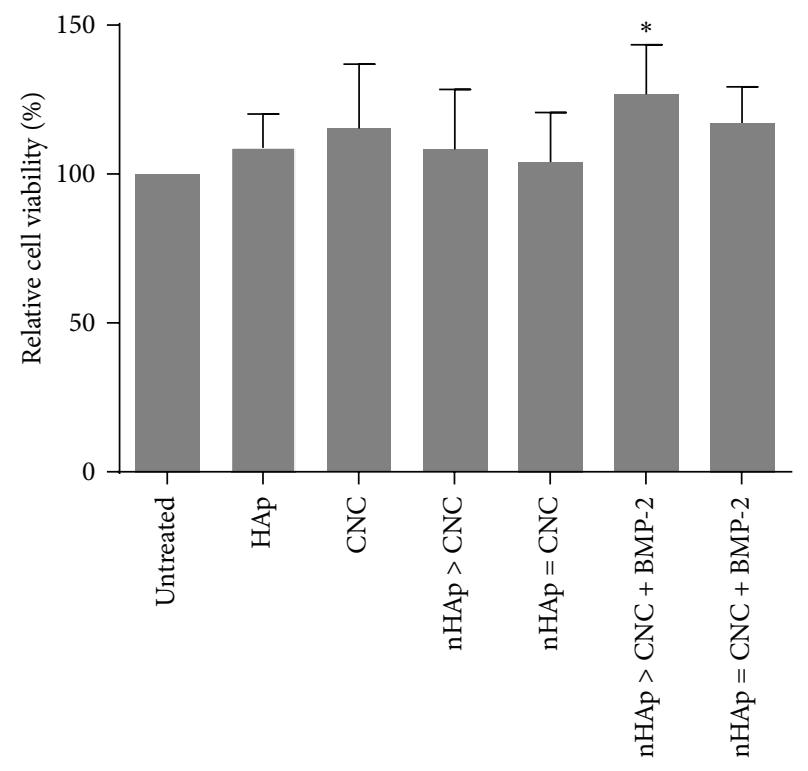

FIGURE 6: Cell viability in human osteoblasts exposed to the different nanoconstructs. The cells were exposed to $1 \mathrm{mg} / \mathrm{mL}$ of HAp, CNC, $\mathrm{Hap}>\mathrm{CNC}, \mathrm{HAP}=\mathrm{CNC}$, Hap $>\mathrm{CNC}+\mathrm{BMP}-2$, and HAP $=\mathrm{CNC}+$ BMP-2 nanoconstructs for $24 \mathrm{~h}$ and cell viability was determined with the MTS reduction assay. Values represent mean \pm standard error of the mean (SEM) from three replicates. An asterisk indicates statistical significance in comparison with the untreated samples $(p<0.05)$.

This result was expected given the fact that one of the reasons HAp was chosen is because of its high porosity in which the pore size of these scaffolds as long as it is equal to or bigger than $20 \mu \mathrm{m}$ will permit the growth of cells [32].

Recent studies have shown that the use of hydroxyapatite(HA-) 2,2,6,6-tetramethylpiperidine-1-oxyl- (TEMPO-) oxidized bacterial cellulose- (TOBC-) GEL composites in calvarial osteoblasts from Sprague-Dawley rats did not compromise the cell viability and promote cell proliferation [33]. Furthermore, other studies have demonstrated that poly-Llactide acid (PLLA)/HA/cellulose composites did not affect the cell viability of rat osteosarcoma cells (URM-106) [34]. In this study we showed that the use of nHAp > CNC with BMP2 , which is an important protein that regulates osteoblast differentiation, promotes the growth of human fetal osteoblast cells (Figure 6). Taken together, these results might suggest that cellulose nanocomposites with the addition of proteins that are important for osteoblast growth and differentiation such as BMP-2 are suitable for biomedical applications in the field of Bone Tissue Engineering.

\section{Conclusion}

The proportion of the $\mathrm{nHAp}=\mathrm{CNC}$ ratio appeared to be the most suitable ratio for this methodology as it provides homogeneity of the particle's size and distribution that was shown by the DLS and SEM analyses. We conclude that the CNC did work as expected by the sol-gel method to include HAp in its network without affecting its degree of 
crystallinity, as shown by the XRD analyses, or the thermal stability as shown by the TGA. Neither of the properties of either CNC or HAp changed due to the chemical interaction between them. The inclusion of BMP-2 does not appear to have a negative effect on the particle size and the charge reduction indicates that there is an interaction between the protein and the composite. The cell proliferation and viability assays demonstrated that all of our composites promoted an increased cell growth, but the nHAp > CNC ratio with BMP2 had a higher growth percentage than the other composites. Therefore it appears to be the best nanocomposite for the fabrication of scaffolds or bioactive layers that can be used in the Bone Tissue Engineering field.

\section{Conflict of Interests}

The authors declare no competing financial interests.

\section{Authors' Contribution}

The paper was written through contributions of all authors. All authors have given approval to the final version of the paper. Claudia S. Herdocia-Lluberes and Simara LaboyLópez contributed equally to this work.

\section{Acknowledgments}

Claudia S. Herdocia-Lluberes is grateful for the support of the UPR-RP Honors Program. The authors acknowledge the UPR Materials Characterization Center (MCC) for the provided support during the attainment of this work. Also, Carlos García and Karlene Vega are acknowledged for their help with all osteoblast cells related experiments. Institutional resources as seed funds were received during the attainment of this work. This work was also supported in part by the NASA Experimental Program to Stimulate Competitive Research (EPSCoR) under Grant no. NNX14AN18A.

\section{References}

[1] H. Liu, H. Peng, Y. Wu et al., "The promotion of bone regeneration by nanofibrous hydroxyapatite/chitosan scaffolds by effects on integrin-BMP/Smad signaling pathway in BMSCs," Biomaterials, vol. 34, no. 18, pp. 4404-4417, 2013.

[2] A. R. Amini, C. T. Laurencin, and S. P. Nukavarapu, "Bone tissue engineering: recent advances and challenges," Critical Reviews in Biomedical Engineering, vol. 40, no. 5, pp. 363-408, 2012.

[3] S. Laï, B. H. Fellah, A. Fatimi et al., "The in vivo degradation of a ruthenium labelled polysaccharide-based hydrogel for bone tissue engineering," Biomaterials, vol. 30, no. 8, pp. 1568-1577, 2009.

[4] S. Bose, M. Roy, and A. Bandyopadhyay, "Recent advances in bone tissue engineering scaffolds," Trends in Biotechnology, vol. 30, no. 10, pp. 546-554, 2012.

[5] N. Pramanik, D. Mishra, I. Banerjee, T. K. Maiti, P. Bhargava, and P. Pramanik, "Chemical synthesis, characterization, and biocompatibility study of hydroxyapatite/chitosan phosphate nanocomposite for bone tissue engineering applications," International Journal of Biomaterials, vol. 2009, Article ID 512417, 8 pages, 2009.

[6] C. W. Hayes and W. F. Conway, "Calcium hydroxyapatite deposition disease," Radiographics, vol. 10, no. 6, pp. 1031-1048, 1990.

[7] H.-K. Ea and F. Lioté, "Advances in understanding calciumcontaining crystal disease," Current Opinion in Rheumatology, vol. 21, no. 2, pp. 150-157, 2009.

[8] G. M. Garcia, G. C. McCord, and R. Kumar, "Hydroxyapatite crystal deposition disease," Seminars in Musculoskeletal Radiology, vol. 7, no. 3, pp. 187-193, 2003.

[9] C. Nguyen, M. Lieberherr, C. Bordat et al., "Intracellular calcium oscillations in articular chondrocytes induced by basic calcium phosphate crystals lead to cartilage degradation," Osteoarthritis and Cartilage, vol. 20, no. 11, pp. 1399-1408, 2012.

[10] S. Facca, D. Lahiri, F. Fioretti et al., "In vivo osseointegration of nano-designed composite coatings on titanium implants," ACS Nano, vol. 5, no. 6, pp. 4790-4799, 2011.

[11] L. L. Hyland, M. B. Taraban, B. Hammouda, and Y. B. Yu, "Mutually reinforced multicomponent polysaccharide networks," Biopolymers, vol. 95, no. 12, pp. 840-851, 2011.

[12] S. C. Chao, M. J. Wang, N. S. Pai, and S. K. Yen, "Preparation and characterization of gelatin-hydroxyapatite composite microspheres for hard tissue repair," Materials Science \& Engineering C: Materials for Biological Applications, vol. 57, pp. 113-122, 2015.

[13] A. Dufresne, "Nanocellulose: a new ageless bionanomaterial," Materials Today, vol. 16, no. 6, pp. 220-227, 2013.

[14] R. M. A. Domingues, M. E. Gomes, and R. L. Reis, “The potential of cellulose nanocrystals in tissue engineering strategies," Biomacromolecules, vol. 15, no. 7, pp. 2327-2346, 2014.

[15] A. Salam, L. A. Lucia, and H. Jameel, "A novel cellulose nanocrystals-based approach to improve the mechanical properties of recycled paper," ACS Sustainable Chemistry and Engineering, vol. 1, no. 12, pp. 1584-1592, 2013.

[16] T. A. Kuriakose, S. N. Kalkura, M. Palanichamy et al., "Synthesis of stoichiometric nano crystalline hydroxyapatite by ethanolbased sol-gel technique at low temperature," Journal of Crystal Growth, vol. 263, no. 1-4, pp. 517-523, 2004.

[17] J. Klinkaewnarong and E. Swatsitang, "Synthesis of nanocrystalline hydroxyapatite by natural biopolymers based Sol-Gel technique," in II. Bio-Materials and Biomimetic Materials, vol. 747 of Advanced Materials Research, pp. 83-86, 2013.

[18] A. K. Nayak, "Hydroxyapatite synthesis methodologies: an overview," International Journal of ChemTech Research, vol. 2, no. 2, pp. 903-907, 2010.

[19] S. S. Lee, B. J. Huang, S. R. Kaltz et al., "Bone regeneration with low dose BMP-2 amplified by biomimetic supramolecular nanofibers within collagen scaffolds," Biomaterials, vol. 34, no. 2, pp. 452-459, 2013.

[20] J. Chłopek, B. Czajkowska, B. Szaraniec, E. Frackowiak, K. Szostak, and F. Béguin, "In vitro studies of carbon nanotubes biocompatibility," Carbon, vol. 44, no. 6, pp. 1106-1111, 2006.

[21] L. P. Zanello, B. Zhao, H. Hu, and R. C. Haddon, "Bone cell proliferation on carbon nanotubes," Nano Letters, vol. 6, no. 3, pp. 562-567, 2006.

[22] S. Baradaran, E. Moghaddam, B. Nasiri-Tabrizi et al., "Characterization of nickel-doped biphasic calcium phosphate/ graphene nanoplatelet composites for biomedical application," Materials Science and Engineering C, vol. 49, pp. 656-668, 2015. 
[23] H. A. Monreal Romero, J. Mora Ruacho, C. A. Martínez Pérez, and P. E. García Casillas, "Synthesis of hydroxyapatite nanoparticles in presence of a linear polysaccharide," Journal of Materials, vol. 2013, Article ID 683268, 5 pages, 2013.

[24] T. Wang, A. Dorner-Reisel, and E. Müller, "Thermogravimetric and thermokinetic investigation of the dehydroxylation of a hydroxyapatite powder," Journal of the European Ceramic Society, vol. 24, no. 4, pp. 693-698, 2004.

[25] C. Echeverria, P. L. Almeida, G. Feio, J. L. Figueirinhas, and M. H. Godinho, "A cellulosic liquid crystal pool for cellulose nanocrystals: structure and molecular dynamics at high shear rates," European Polymer Journal, vol. 72, pp. 72-81, 2015.

[26] Y. Tang, X. Shen, J. Zhang, D. Guo, F. Kong, and N. Zhang, "Extraction of cellulose nano-crystals from old corrugated container fiber using phosphoric acid and enzymatic hydrolysis followed by sonication," Carbohydrate Polymers, vol. 125, pp. 360-366, 2015.

[27] A. M. de Araujo Junior, G. Braido, S. Saska et al., "Regenerated cellulose scaffolds: preparation, characterization and toxicological evaluation," Carbohydrate Polymers, vol. 136, pp. 892-898, 2016.

[28] M. L. Macdonald, R. E. Samuel, N. J. Shah, R. F. Padera, Y. M. Beben, and P. T. Hammond, "Tissue integration of growth factor-eluting layer-by-layer polyelectrolyte multilayer coated implants," Biomaterials, vol. 32, no. 5, pp. 1446-1453, 2011.

[29] Z. Lu, C. Huangfu, Y. Wang et al., "Kinetics and thermodynamics studies on the BMP-2 adsorption onto hydroxyapatite surface with different multi-morphological features," Materials Science \& Engineering C: Materials for Biological Applications, vol. 52, pp. 251-258, 2015.

[30] M. Ventura, O. C. Boerman, G. M. Franssen, E. Bronkhorst, J. A. Jansen, and X. F. Walboomers, "Monitoring the biological effect of BMP-2 release on bone healing by PET/CT," Journal of Controlled Release, vol. 183, pp. 138-144, 2014.

[31] X. Li, H. Gao, M. Uo et al., "Maturation of osteoblast-like SaoS2 induced by carbon nanotubes," Biomedical Materials, vol. 4, no. 1, Article ID 015005, 2009.

[32] L. Kong, Y. Gao, W. Cao, Y. Gong, N. Zhao, and X. Zhang, "Preparation and characterization of nano-hydroxyapatite/chitosan composite scaffolds," Journal of Biomedical Materials Research Part A, vol. 75, no. 2, pp. 275-282, 2005.

[33] M. Park, D. Lee, S. Shin, and J. Hyun, "Effect of negatively charged cellulose nanofibers on the dispersion of hydroxyapatite nanoparticles for scaffolds in bone tissue engineering," Colloids and Surfaces B: Biointerfaces, vol. 130, pp. 222-228, 2015.

[34] S. Eftekhari, I. El Sawi, Z. S. Bagheri, G. Turcotte, and H. Bougherara, "Fabrication and characterization of novel biomimetic PLLA/cellulose/hydroxyapatite nanocomposite for bone repair applications," Materials Science and Engineering C, vol. 39, no. 1, pp. 120-125, 2014. 

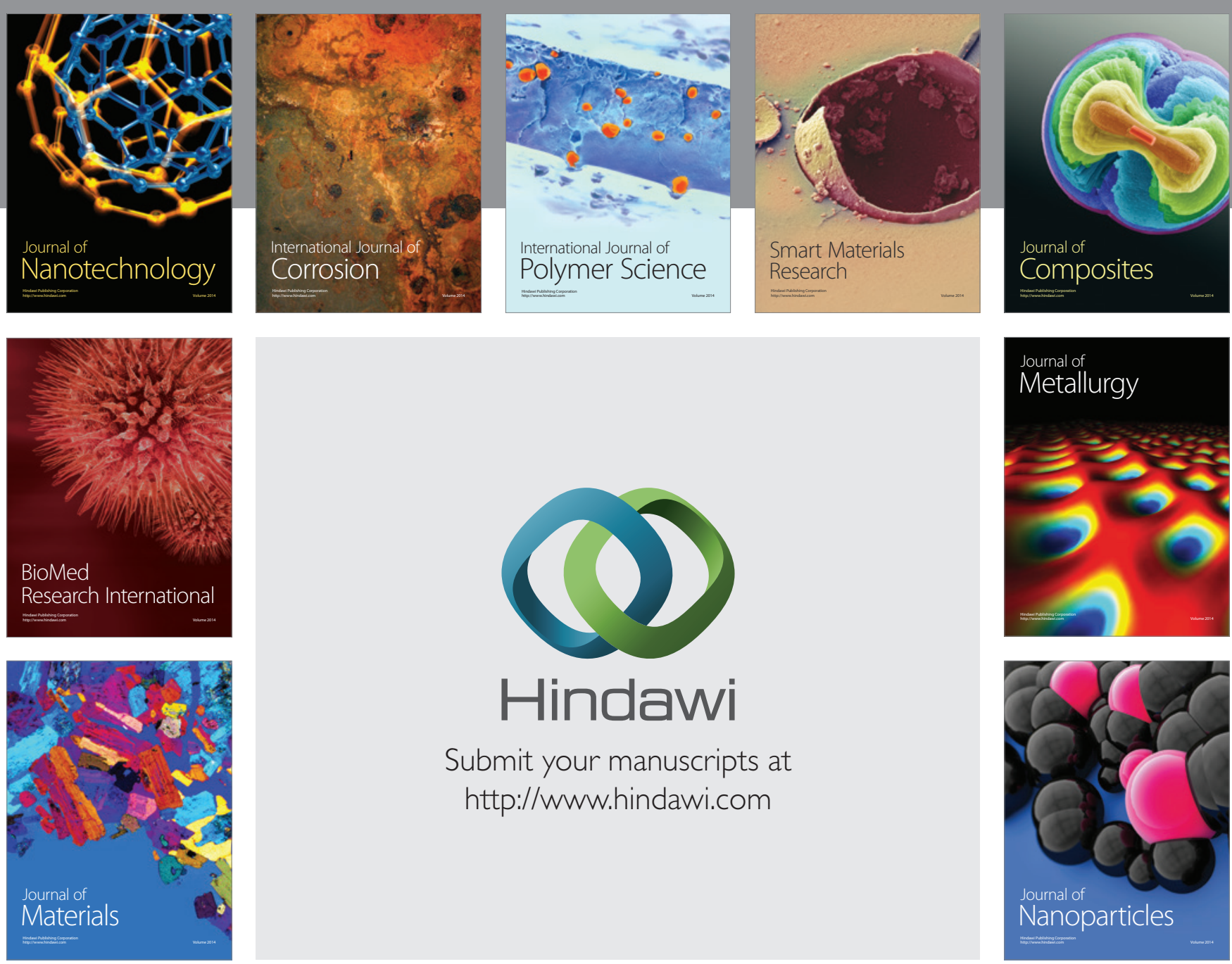

Submit your manuscripts at http://www.hindawi.com
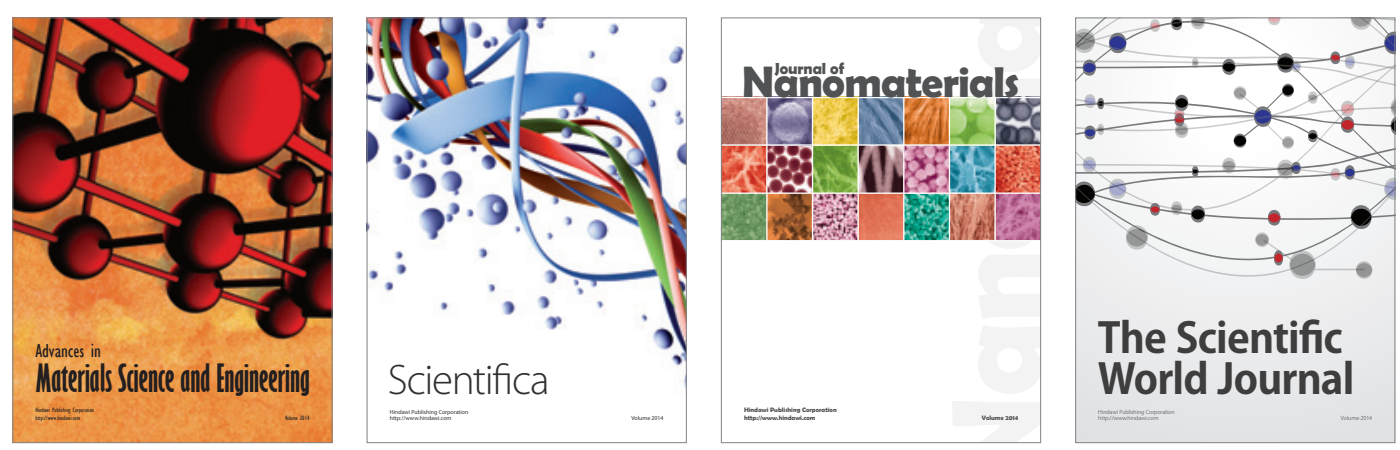

\section{The Scientific World Journal}
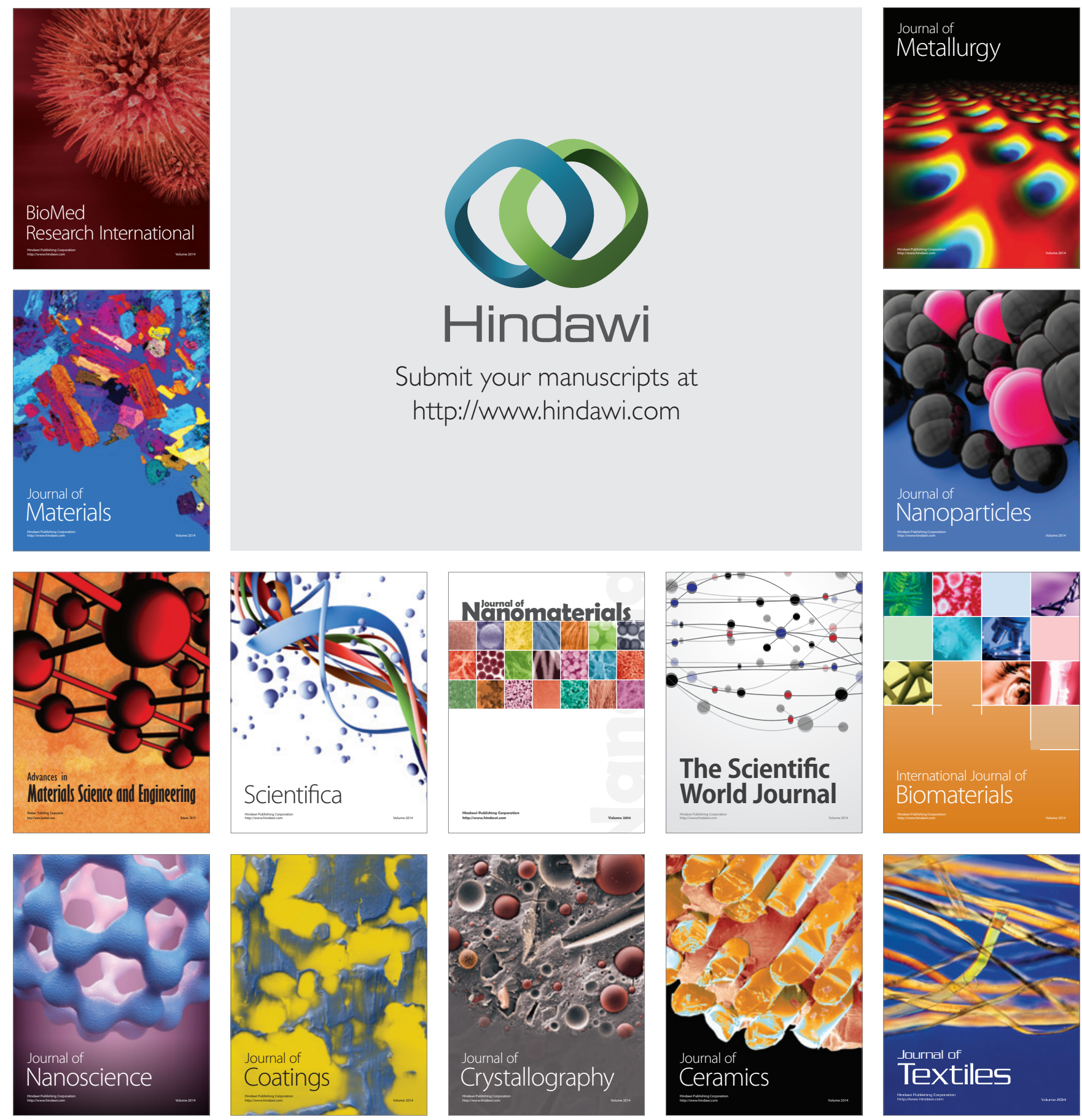